\title{
Recherches sur la survivance dans le milieu marin de diverses souches de Levures isolées de l'Homme
}

\author{
par M. LAHOURCADE *
}

Depuis les travaux précurseurs de B. Fischer en 1894 (7), un certain nombre de prospections microbiologiques marines intéressant les principaux océans et mers du globe, ont été réalisées au cours des années récentes $(1,4,5,6,10,11,15,16,17)$. Leurs résultats démontrent la présence constante dans le milieu marin de levures appartenant à des espèces variées, assez souvent identiques ou apparentées à celles que l'on rencontre habituellement en milieu terrestre.

Cependant, la répartition de ces levures n'apparaît pas uniforme : d'une manière générale, leur concentration diminue du littoral vers le large ainsi que de la surface vers les profondeurs, l'étage planctonique leur étant, semble-t-il, particulièrement favorable.

D'autre part, la densité de ces levures, le long des côtes très peuplées, peut atteindre des chiffres élevés: souvent plus de mille par litre sur les côtes de Floride (14), quelques centaines dans une grande mer intérieure comme la mer Noire (10); quant aux recherches que nous poursuivons actuellement dans le bassin d'Arcachon, baie marine de dimension relativement réduite pour une population riveraine importante, notamment en période estivale, elles nous ont permis de constater des densités encore plus fortes, atteignant fréquemment en été 2.000 levures par litre à marée haute, mais jusqu'à plus de 10.000 parfois à marée basse (11).

Or, la présence constante et nombreuse des levures dans l'eau de mer, jointe à leur répartition peu homogène, posent actuellement un problème difficile à résoudre et dont la solution intéresse cependant directement, non seulement l'hygiène générale et la santé publique, mais aussi l'équilibre biologique du milieu marin dont nous aurions le plus grand intérêt à sauvegarder l'intégrité. Il s'agit de préciser en effet, l'origine exacte de ces levures « marines » :

- appartiennent-elles spécifiquement au milieu marin, dont elles forment un élément constitutif normal, naturel, incapable de venir perturber aujourd'hui un équilibre biologique établi depuis des millénaires?

- ou bien, au contraire, ces levures dites « marines » ne sont-elles pas plutôt des levures terrestres, introduites dans le milieu marin avec les eaux de ruissellement, et sur-

(*) Ce travail a fait l'objet d'une communication à la Société française de Mycologie médicale, réunie en Congrès à Montpellier, le 7 mai 1966. 
tout maintenant avec les eaux usées déversées de plus en plus généreusement par les égouts, soit dans les fleuves, soit directement sur le littoral.

Il est à craindre malheureusement que cette dernière hypothèse, corroborée par les constatations précédentes, ne corresponde à la réalité : or, si beaucoup de levures terrestres sont saprophytes et commensales, nous savons que certaines sont aussi pathogènes pour les végétaux, les animaux terrestres et pour l'homme; introduites de plus en plus massivement dans le milieu marin, ne risquent-elles pas d'y créer bientôt des déséquilibres biologiques importants, par la contamination de la flore et de la faune marines, causant notamment des maladies des mollusques, des crustacés et des poissons comestibles. D'ailleurs, à diverses reprises, des auteurs japonais et américains $(8,9,12,13)$ ont isolé des levures (notamment des Rhodotorula) qu'ils jugeaient responsables d'altération des huîtres, des coquillages, des crevettes, etc...

On peut aborder le problème des origines et de la spécificité des levures « marines $\gg$ de deux façons :

La première consisterait, par des prospections systématiques nombreuses, échelonnées dans l'espace et dans le temps, à surveiller les variations éventuelles de la densité des levures marines. Si cette densité s'avérait alors demeurant à un niveau à peu près constant, partout, en dépit de la croissance des apports d'eaux usées et de la souillure des eaux côtières dans les stations balnéaires, il faudrait admettre que les levures marines sont réellement spécifiques et que les levures exogènes, terrestres, incapables de s'adapter au milieu marin, y disparaissent rapidement. Malheureusement, cette surveillance de la densité des levures « marines » par des prospections microbiologiques systématiques (actuellement entreprises dans quelques stations de Biologie Marine) demeure une entreprise difficile, de longue haleine, exigeant d'ailleurs un personnel technique qualifié et un matériel plus importants que ceux dont nous disposons actuellement.

C'est pourquoi, nous avons pensé qu'il serait possible de contribuer plus simplement à l'étude de cette question, en observant directement le comportement de levures terrestres plongées expérimentalement dans un milieu marin, en s'écartant aussi peu que possible des conditions naturelles.

\section{Technique de l'expérimentation}

Nous avons choisi 15 souches de levures, appartenant à des espèces différentes des genres Candida, Torulopsis, Cryptococcus, Saccharomyces et Rhodotorula, toutes ces souches ayant été isolées à partir de prélèvements bronchoscopiques humains provenant du Centre anticancéreux de Bordeaux.

Le milieu marin dans lequel nous les avons introduites, était constitué par de l'eau de mer fraîchement prélevée dans le Bassin d'Arcachon et débarrassée par une filtration immédiate, sur membrane cellulosique ultrafiltrante, de toute particule organique allant jusqu'aux bactéries. 
- Pour chaque souche étudiée, le volume d'une colonie de $1 \mathrm{~cm}$ de diamètre, entretenue sur milieu de Sabouraud, a été introduite dans un ballon contenant $200 \mathrm{ml} \mathrm{du}$ milieu marin ultrafiltré : la concentration était ainsi approximativement de l'ordre de 5.000 levures par millilitre.

- Chaque mois, nous avons procédé aux manipulations suivantes pour chacun des 15 ballons ensemencés :

$1^{\circ}$ Un renouvellement partiel du milieu de culture, afin de pallier son épuisement par la levure, mais aussi d'éliminer dans une certaine mesure les substances dissoutes résultant de l'autolyse des levures mortes, substances auxquelles on pourrait attribuer un rôle non négligeable dans la survie des levures restantes. Pour cela, nous avons aspiré par pipetage en surface la moitié du milieu $(100 \mathrm{ml})$, en prenant de grandes précautions pour ne pas disperser les levures qui se trouvaient en majorité déposées au fond du ballon.

$2^{\circ}$ Ayant dispersé ensuite par une agitation douce ces levures, nous avons prélevé dix gouttes de cette suspension dans chaque ballon, avec une pipette compte-gouttes, et les avons déposées à la surface de boîtes de Pétri contenant un milieu de Sabouraud glucosé.

$3^{\circ}$ Le contenu des ballons était recomplété finalement à $200 \mathrm{ml}$ par addition d'eau de mer fraîchement prélevée et ultrafiltrée.

$4^{\circ}$ Le développement des levures était surveillé quotidiennement dans les 15 boîtes de Pétri, et l'intensité de ce développement apprécié en tenant compte de son caractère :

- soit homogène: la goutte déposée formant d'emblée une tache crémeuse uniforme, ce que nous avons noté par ++++ ;

- soit hétérogène : la goutte déposée se manifestant au début par l'apparition de colonies distinctes plus ou moins nombreuses; lorsque ces colonies séparées étaient très nombreuses dans chacune des 10 gouttes déposées, nous avons noté +++ ; si ces colonies étaient peu nombreuses dans chaque goutte, mais leur nombre total dans la boîte encore supérieur à 20 colonies : ++ ; si par contre, le nombre total des colonies dans la boîte était inférieur à 20 , nous avons noté le phénomène par une + .

$5^{\circ}$ Nous avons procédé à un contrôle d'identité de la levure obtenue en culture dans chaque boîte, pour être sûr de l'absence dans les ballons de toute souillure exogène qui aurait pu éventuellement troubler les résultats. D'ailleurs, les grandes précautions d'asepsie observées au cours de toutes ces manipulations, nous ont permis d'éviter cet écueil.

\section{Résultats}

Les résultats obtenus au cours de cette expérience sont réunis Jans le tableau cicontre :

Il en ressort immédiatement que toutes les espèces de levures expérimentées ont manifesté une survie égale ou supérieure à deux mois dans l'eau de mer ultrafiltrée : on peut en déduire que la composition chimique de cette dernière, en particulier sa forte salinité $(35 \%)$, ne semble en aucune façon constituer un obstacle majeur à la survie des levures terrestres: seul, le degré de leurs exigences nutritionnelles en substances organiques complexes, paraît en définitive, régler la durée de leur survie dans le milieu marin.

Les levures dont la courte survie n'excède pas quatre mois: Torulopsis sphaerica, Candida pseudotropicalis, Candida azymatique, Saccharomyces lactis, C. krusei et Rho dotorula glutinis, exigent certainement la présence dans le milieu marin, de ces subs- 


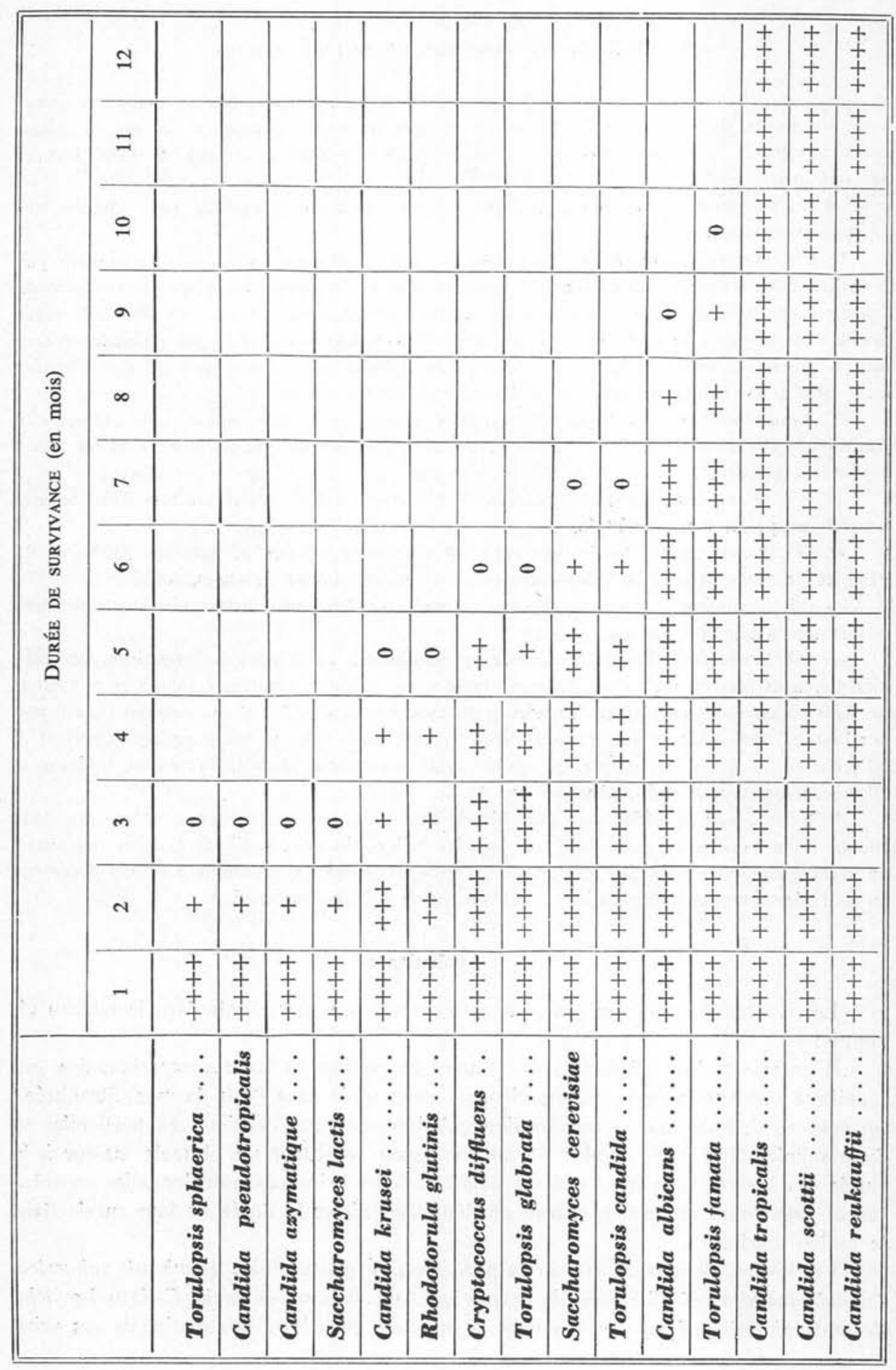


tances organiques complexes, qui leur ont été fournies ici sans doute exclusivement par l'autolyse des levures mortes, et qu'elles ont de ce fait rapidement épuisées, compte tenu du renouvellement partiel, mensuel du milieu.

Par contre, les exigences nutritionnelles de Cryptococcus diffluens, T. glabrata, $S$. cerevisiae, $T$. candida, $C$. albicans et $T$. famata, dont la survie fut de l'ordre de cinq à neuf mois, apparaissent ici plus mesurées; il est permis de penser que ces levures, plongées dans le milieu marin naturel, pourraient y survivre plus longtemps et même s'y adapter définitivement, dans la mesure où elles y rencontreraient un substratum organique particulaire, mort ou vivant, aux dépens duquel elles pourraient se développer à l'état saprophyte ou commensal, voire même en éléments parasites.

Quant à Candida albicans, levure pathogène que l'on peut isoler en période estivale, le long des côtes où se manifestent d'importantes activités balnéaires (nous l'avons trouvée à deux reprises, en août 1965, dans l'eau littorale en face de la plage d'Arcachon), sa survie de huit mois constatée au cours de notre expérience, suffit certainement pour expliquer la pathogénie de ces petites épidémies de Candidoses cutanées et ano-génitales qui fleurissent chaque année, notamment chez les estivants assidus de ces plages « à la mode » où la foule s'entasse dans la pire des promiscuités et sans respecter souvent les règles les plus élémentaires de l'hygiène corporelle.

Les trois dernières espèces enfin : $C$. tropicalis, $C$. scottii et $C$. reukauffii, ont fait la preuve ici de leur adaptation complète et apparemment définitive au milieu marin, quelque soit la teneur de ce dernier en particules organisées. Il est d'ailleurs remarquable que l'une de celles-ci, $C$. tropicalis, est l'espèce la plus communément et la plus universellement isolée du milieu marin, lors des prospections microbiologiques actuelles.

\section{Conclusion}

Notre modeste expérience, bien que critiquable sans doute dans le détail de certaines de ses modalités, apporte certainement une preuve supplémentaire que la pollution microbiologique croissante du milieu marin, tant par les eaux usées déversées directement dans les fleuves ou sur les côtes, que par la souillure des plages et des eaux littorales dans les stations balnéaires, constitue un danger réel pour le maintient de l'équilibre biologique naturel de la flore et de la faune marines.

Elle prouve en effet, que les levures, au même titre que les bactéries $(2,3)$ et à un degré égal sinon supérieur, sont capables de s'intégrer rapidement dans un nouveau milieu biologique, même très différent par ses composantes physico-chimiques de leur milieu initial, naturel ; leurs grandes facultés d'adaptation au commensalisme puis à l'état parasitaire, font donc courir le risque d'une contamination de la flore et la faune marine, contamination dont l'importance et la gravité des conséquences demeurent imprévisibles, en l'état de nos connaissances actuelles. 
Il nous paraît opportun de rappeler d'ailleurs, à l'occasion, les troublantes constatations faites récemment par N. Van Uden et R. Castelo-Branco (14) et qui rejoignent les nôtres: ces auteurs ont en effet rencontré en très grande abondance à La Jolla, sur la côte de Californie, deux levures ascosporées d'espèces très voisines et particulièrement originales (bien caractérisées par leurs ascospores en aiguille, uniques dans un asque claviforme); il s'agit de Metschnikowiella zobellii qu'ils ont isolée de l'eau de mer, de l'intestin de divers poissons ainsi que de la surface d'algues géantes; et de Metschnikowiella krissii, qu'ils ont isolée de l'eau de mer seulement.

Or, en 1864, le savant russe Metschnikoff avait observé et décrit à Moscou, une levure morphologiquement identique qu'il nommait à l'époque Monospora bicuspidata, parasitant abondamment la cavité générale de Daphnia magna, petit crustacé d'eau douce très commun dont elle provoquait la mort : il est très remarquable que Van Uden et Castelo-Branco aient pu vérifier expérimentalement que leurs deux Metschnikowiella marines étaient parfaitement capables non seulement de s'adapter à l'eau douce, mais aussi d'y parasiter mortellement Daphnia Magna.

Il est probable que des investigations microbiologiques plus poussées du monde marin, de sa faune et de sa flore, multiplieront dans l'avenir les exemples de ce genre et confirmeront le bien-fondé de nos craintes actuelles.

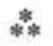

Il serait donc souhaitable que les Pouvoirs publics prennent conscience du développement actuel d'une pollution microbiologique des eaux douces et marines qui, sans être aussi spectaculaire dans ses effets immédiats que la pollution chimique, n'en constitue pas moins, à long terme, une menace sérieuse pour l'équilibre biologique du monde marin, dont l'homme demeurera plus que jamais tributaire pour son alimentation et sa santé. Aussi, serait-il assez urgent de mettre à l'étude des techniques d'épuration simples, afin de réduire au maximum dans l'avenir, la pollution microbiologique des mers et des océans par les eaux usées.

Il serait enfin nécessaire d'informer le grand public, au moyen de campagnes sanitaires largement diffusées, que l'eau de mer ne possède aucune des vertus purificatrices «quasi-antibiotiques » qui lui sont attribuées trop souvent; qu'il y va de l'intérêt général et de chacun, de traiter médicalement jusqu’à leur stérilisation, toutes les lésions ouvertes, de nature microbienne ou parasitaire fongique, avant de se livrer à la pratique de la balnéation en eau libre, douce ou marine.

Les médecins et les hygiénistes ont, en l'occurence, un rôle très important à jouer.

\section{Résumé}

Dans le but d'apprécier expérimentalement les facultés adaptatives éventuelles des levures terrestres introduites dans le milieu marin, 15 espèces de levures différentes, isolées de prélèvements endobronchiques humains, ont été cultivées pendant un an dans de l'eau de mer purifiée par ultrafiltration; mensuellement, ce milieu est renouvelé 
par moitié et la survivance de la levure vérifiée par rétroculture. Les durées de survie ont été pour Torulosis sphaerica, Candida pseudotropicalis, C. azymatique, Saccharomyces lactis : deux mois; C. krusei, Rhodotorula glutinis : quatre mois ; Cryptococcus diffluens, $T$. glabrata: cinq mois ; $S$. cerevisiae et $T$. candida: six mois; $C$. albicans : huit mois ; $T$. famata: neuf mois ; $C$. tropicalis, $C$. scottii, $C$. reukauffii : plus de douze mois. Ces résultats prouvent les grandes facultés adaptatives des levures et leur peu de spécificité pour le milieu marin ou terrestre. Ils confirment donc le danger constitué par la pollution microbiologique croissante des eaux fluviales et côtières, menaçant l'équilibre biologique marin, l'hygiène générale et la santé humaine.

\section{Bibliographie}

1. Bhat (J. V.) et Kachwalla (N.), 1955. - Marine yeasts of the Indian Coast. Proc. indian Acad. Sci., sect. B, 41, 9-15.

2. Brisou (J.), 1960. - Microflore d'un lac salé saharien. Ann. Inst. Pasteur, 99, 450-3.

3. Brisou (J.), Vargues (H.) et Cadeillan (J.), 1961. - Vie des microbes terrestres en milieu marin. Action des composés iodés. C.R. Soc. Biol., 155, 887-90.

4. CAPriotti (A.), 1962. - Yeasts of the Miami, Florida, area. III. From sea water, marine animals and decaying materials. Arch. Mikrobiol., 42, 407-14.

5. Fell (J. W.), Ahearn (D. G.), Meyers (S. P.), Roth (F. J.), 1960. - Isolation of yeasts from Biscayne Bay, Florida, and adjacent benthic areas. Limnol. Oceanogr., 5, 366-71.

6. Fell (J. W.), 1961. - A new species of Saccharomyces isolated from subtropical estuary, Antonie Leeuwenhoek, 27, 27-30.

7. Fischer (B.), 1894. - Die Bakterien des Meers nach den Untersuchungen der Plankton Expedition unter gleichzeitiger Berucksichtigung einiger älterer und neuerer Untersuchungen. Ergebnisse der Plankton-Expedition der Humboldt-Stiftung, 4, 1-83.

8. Hunter (A. C.), 1920. - A pink yeast causing spoilage in oysters. U.S. Dept. Agric. Bull., $\mathrm{n}^{\circ} 819,1-24$.

9. Kobayashi (Y.), Tsubaki (K.), Soneda (M.), 1953. - Marine yeasts isolated from little neck clam. Bull. Nat. Sci. Mus. Tokyo, 33, 47-52.

10. Kriss (A. E.), Nowoshiloff (M. I.), 1954. - Are yeasts organisms inhabitants of seas and oceans. Mikrobiologiia, 23, 669-683. Traduit du russe par J. S. Zobell, Scripps Institution of Oceanography, University of California, La Jolla, U.S.A.

11. Lahourcade (M.) et Brisou (J.). - Les levures marines du Bassin d'Arcachon. I. Etude systématique des levures isolées des eaux côtières (public. en cours).

12. Mc Cormack (G.), 1950. - Pink yeast isolated from oysters grown at temperatures below freezing. Fish and Wildlife Service U.S. Dept. Interior, 12, II A.

13. Phaff (H. J.), Mrak (E. M.), Williams (O. B.), 1952. - Yeasts isolated from shrimp. Mycologia, 44, 431-51. 
14. Van Uden (N.), Castelo-Branco (R.), 1961. - Metschnikowiella zobelli sp. nov., and M. krissii sp. nov., two yeasts from the Pacific Ocean pathogenic for Daphnia magna. J. gen. Microbiol., 26, 141-8.

15. VAN Uden (N.), KolipinSKI (M. C.), 1962. - Torulopsis haemulonii nov. spec., a yeast from the Atlantic Ocean. Antonie Leeuwenhoeck, 28, 78-80.

16. VAN Uden (N.), Zobell (C. E.), 1962. - Candida marina nov. spec., Torulopsis torresii nov. spec. and T. maris nov. spec., three yeasts from the Torres Strait. Antonie Leeuwenhoeck, 28, 275-283.

17. ZoBell (C. E.), 1946. - Marine Microbiology. Chronica Botanica Company. Waltham Mass.

Station de Biologie Marine d'Arcachon

Laboratoires de Parasitologie de Poitiers et de Bordeaux 